\title{
Critical periods in which dopamine exerts control over behavioral responding during Pavlovian learning
}

Merridee J. Lefner ${ }^{1}$, Claire E. Stelly ${ }^{1}$, Kaitlyn M. Fonzi ${ }^{1}$, Hector Zurita ${ }^{1}$, and Matthew J. Wanat ${ }^{1}$

${ }^{1}$ Neurosciences Institute and Department of Biology, University of Texas at San Antonio, San Antonio, TX, USA.

\section{Corresponding Author:}

Matthew J. Wanat

Neurosciences Institute

Department of Neuroscience, Developmental and Regenerative Biology

University of Texas at San Antonio

One UTSA Circle

San Antonio, TX 78249

Matthew.wanat@utsa.edu

210.458 .6684 


\begin{abstract}
:
Learning the association between rewards and predictive cues is critical for appetitive behavioral responding. The mesolimbic dopamine system is thought to play an integral role in establishing these cue-reward associations. The dopamine response to cues can signal differences in reward value, though this emerges only after significant training. This suggests that the dopamine system may differentially regulate behavioral responding depending on the phase of training. To address this, separate groups of male rats were trained on Pavlovian tasks in which distinct audio cues signaled either differences in reward size or differences in reward rate. The dopamine receptor antagonist flupenthixol was systemically administered prior to either the first ten sessions of training (acquisition phase) or the second ten sessions of training (expression phase) and we monitored the effect of these manipulations for an additional ten training sessions. We identified acute effects of dopamine receptor antagonism on conditioned responding, the latency to respond, and post-reward head entries in both Pavlovian tasks. Interestingly, dopamine receptor antagonism during the expression phase produced persistent deficits in behavioral responding only in rats trained on the reward size Pavlovian task. Together, our results illustrate that dopamine's control over behavior in Pavlovian tasks depends upon one's prior training experience and the information signaled by the cues.
\end{abstract}

\title{
Introduction:
}

Learning to associate rewarding outcomes to the cues that predict them is a critical process for promoting efficient reward-seeking behavior. These cue-reward associations are regulated by the mesolimbic dopamine system (Phillips et al., 2007; Salamone and Correa, 2012). Prior studies illustrate that dopamine signaling can influence the acquisition and expression of conditioned responding in appetitive Pavlovian tasks (Di Ciano et al., 2001; Flagel et al., 2011; Wassum et al., 2011; Saunders and Robinson, 2012; Sculfort et al., 2016; Fraser and Janak, 2017; Roughley and Killcross, 2019; Stelly et al., 2020; Stelly et al., 2021). For example, antagonizing dopamine receptors during early training sessions acutely impairs conditioned responding (Di Ciano et al., 2001; Flagel et al., 2011; Sculfort et al., 2016; Roughley and Killcross, 2019; Stelly et al., 2021). These deficits in conditioned responding can persist beyond 
the sessions in which the dopamine receptor antagonist was administered, which illustrates dopamine signaling is critical for reward learning (Flagel et al., 2011; Sculfort et al., 2016; Roughley and Killcross, 2019; Stelly et al., 2020; Stelly et al., 2021). In well-trained animals, antagonizing dopamine receptors or optogenetic inhibition of dopamine neurons acutely suppresses conditioned responding (Di Ciano et al., 2001; Saunders and Robinson, 2012; Fraser and Janak, 2017; Heymann et al., 2020; Lee et al., 2020; Morrens et al., 2020). However, it is unclear if perturbations of the dopamine system in well-trained animals can produce persistent alterations in behavior.

The reward delivery evokes a pronounced elevation in dopamine transmission during early Pavlovian training (Schultz et al., 1997; Day et al., 2007; Coddington and Dudman, 2018). After the subject experiences multiple cue-reward pairings, the reward-evoked dopamine response decays and the cue-evoked dopamine response increases (Schultz et al., 1997; Day et al., 2007; Coddington and Dudman, 2018). In well-trained animals, dopamine neurons respond to cues to encode differences in reward-related information including reward size (Tobler et al., 2005; Roesch et al., 2007; Gan et al., 2010; Lefner et al., 2022), reward probability (Fiorillo et al., 2003; Hart et al., 2015), and reward rate (Fonzi et al., 2017; Stelly et al., 2021). However our recent work demonstrates that cue-evoked dopamine release does not signal differences in reward value during early training sessions, though these value signals emerge after extended training (Fonzi et al., 2017; Stelly et al., 2021; Lefner et al., 2022). These findings suggest that the dopamine system may differentially regulate behavioral responding depending on whether the subject is learning the task or is well-trained.

In this study, we trained separate groups of rats on two different Pavlovian tasks that elicit goal-tracking behavior. In the Pavlovian Reward Size task, two distinct audio cues signaled the delivery of either a small reward or a large reward (Lefner et al., 2022). In the Pavlovian Reward Rate task, two distinct audio cues both signaled the delivery of a small reward but differed in the time elapsed since the previous reward delivery (i.e. reward rate) (Fonzi et al., 2017; Stelly et al., 2021). The dopamine receptor antagonist flupenthixol was systemically administered during different phases of training to determine when the dopamine system regulates behavioral responding in these Pavlovian tasks. Rats received flupenthixol injections 
prior to either the first ten sessions of training (Acquisition phase) or the second ten sessions of training (Expression phase) and we monitored the effect of these manipulations for an additional ten training sessions. We quantified the effect of dopamine receptor antagonism on behavioral responding during the cue (e.g. conditioned responding and latency to respond) as well as the number of head entries following the reward delivery. Across Pavlovian tasks, flupenthixol treatment acutely impaired conditioned responding, the latency to respond, and post-reward head entries. However, dopamine receptor antagonism produced persistent deficits in behavioral responding exclusively during the Pavlovian Reward Size task. These findings highlight critical periods in which dopamine controls behavior in a manner that depends on the reward-related information signaled by the cues.

\section{Methods:}

Subjects and surgery

All procedures were approved by the Institutional Animal Care and Use Committee at the University of Texas at San Antonio. Male Sprague-Dawley rats (Charles River, MA) weighing 300-350 g were pair-housed upon arrival and given ad libitum access to water and chow and maintained on a 12-hour light/dark cycle.

\section{Behavioral procedures}

One week after arrival, rats were placed and maintained on mild food restriction $(\sim 15 \mathrm{~g} /$ day of standard lab chow) to target $90 \%$ free-feeding weight, allowing for an increase of $1.5 \%$ per week. Behavioral sessions were performed in chambers (Med Associates) that had grid floors, a house light, a food tray, a food tray light, and two auditory stimulus generators $(4.5 \mathrm{kHz}$ tone, and either $2.5 \mathrm{kHz}$ tone or white noise). To familiarize rats with the chamber and food retrieval, rats underwent a single magazine training session in which 20 food pellets (45 mg, BioServ) were non-contingently delivered at a $90 \pm 15 \mathrm{~s}$ variable interval. Rats then underwent 30 sessions (1/day) of Pavlovian training under the Pavlovian Reward Size or Pavlovian Reward Rate tasks as described previously (Fonzi et al., 2017; Stelly et al., 2021; Lefner et al., 2022). 
Training sessions for the Pavlovian Reward Size task consisted of 50 trials where the termination of a $5 \mathrm{~s}$ audio cue $(\mathrm{CS} ; 2.5 \mathrm{kHz}$ tone or $4.5 \mathrm{kHz}$ tone, counterbalanced across animals) resulted in the delivery of a single food pellet (Small Reward trial) or three food pellets (Large Reward trial) and illumination of the food port light for $4.5 \mathrm{~s}$. Each session contained 25 Small Reward trials and 25 Large reward trials delivered in a pseudorandom order, with a $45 \pm 5$ s ITI between all trials. Training sessions for the Pavlovian Reward Rate task consisted of 50 trials where the termination of a $5 \mathrm{~s}$ audio cue (white noise or $4.5 \mathrm{kHz}$ tone, counterbalanced across animals) resulted in the delivery of a single food pellet and illumination of the food port light for $4.5 \mathrm{~s}$. Each session contained 25 High Rate trials in which the CS was presented after a $20 \pm 5 \mathrm{~s}$ ITI, and 25 Low Rate trials in which the CS was presented after a $70 \pm 5 \mathrm{~s}$ ITI, delivered in a pseudorandom order. Conditioned responding was quantified as the change in the rate of head entries during the $5 \mathrm{~s}$ CS relative to the 5 s preceding the CS delivery (Fonzi et al., 2017; Stelly et al., 2021; Lefner et al., 2022). We also quantified the latency to initiate a head entry during the CS. For the post-US analysis, we calculated the number of head entries made during the $9 \mathrm{~s}$ following reward delivery. This $9 \mathrm{~s}$ post-reward window corresponds to $4.5 \mathrm{~s}$ in which the tray light in the food port was illuminated and an equivalent $4.5 \mathrm{~s}$ after the tray light turned off.

\section{Pharmacology}

Flupenthixol dihydrochloride (Tocris) was dissolved in sterile $0.9 \% \mathrm{NaCl}$. Rats received i.p. injections of flupenthixol $(225 \mathrm{ug} / \mathrm{kg})$ or saline vehicle 1 hour prior to Pavlovian training sessions (Flagel et al., 2011). Flupenthixol injections were administered prior to sessions 1-10 (Acquisition phase) or prior to sessions 11-20 (Expression phase). No injections were administered prior to sessions 21-30. The group sizes are as follows: Pavlovian Reward Size groups: $n=8$ control saline, 8 Acquisition phase flupenthixol, 8 Expression phase flupenthixol; Pavlovian Reward Size groups: $n=10$ control saline, 10 Acquisition phase flupenthixol, 10 Expression phase flupenthixol.

\section{Data analysis}


Statistical analyses were performed in GraphPad Prism 9. Behavioral responding was analyzed using a mixed-effects model fit (restricted maximum likelihood method), repeated measures where appropriate. The Geisser-Greenhouse correction was applied to address unequal variances between groups where applicable. Note that data are plotted separately by trial type for visual clarity in all figures. The full list of statistical analyses are presented in Supplementary Table 1.

\section{Results:}

Separate groups of rats were trained on two different Pavlovian tasks in which distinct audio cues signaled different reward values. The Reward Size Pavlovian task utilized one audio cue (CS) that signaled the delivery of a single sucrose pellet (US; Small Reward trial) and a distinct audio cue that signaled the delivery of three sucrose pellets (Large Reward trial, Fig. 1A). The Reward Rate Pavlovian task used two distinct $5 \mathrm{~s}$ audio cues (CS) that both signaled the delivery of a single sucrose pellet (US), but the CSs differed in the time elapsed since the previous reward (Fonzi et al., 2017; Stelly et al., 2021). In High Rate trials the CS was presented $20 \pm 5 \mathrm{~s}$ ITI following the previous reward delivery, and in Low Rate trials the CS was presented $70 \pm 5$ s following the previous reward delivery (Fig. 2A). To determine how the dopamine system regulates behavioral responding across different phases of training, we systemically administered the non-selective dopamine receptor antagonist flupenthixol one hour prior to the first ten training sessions (Acquisition phase) or the next ten training sessions (Expression phase). Control groups were given saline injections for the first twenty sessions. All rats received no injections during the final ten training sessions.

In the Pavlovian Reward Size task, there was no difference in conditioned responding between Small and Large Reward trials (Sessions 1-10 three-way mixed-effects analysis; reward size effect: $F_{(1,14)}=2.52, p=0.14$; Fig. 1B), consistent with prior research (Lefner et al., 2022). Antagonizing dopamine receptors during the Acquisition phase impaired conditioned responding in both trial types (Sessions 1-10 three-way mixed-effects analysis; treatment effect: $F_{(1,126)}=$ 9.27, $p=0.003$; session $\mathrm{x}$ treatment effect: $F_{(9,126)}=2.53, p=0.01$; Fig. 1B). Conditioned responding remained lower in rats that had previously received flupenthixol during the 
A

Pavlovian Reward Size task (50 trials per session)
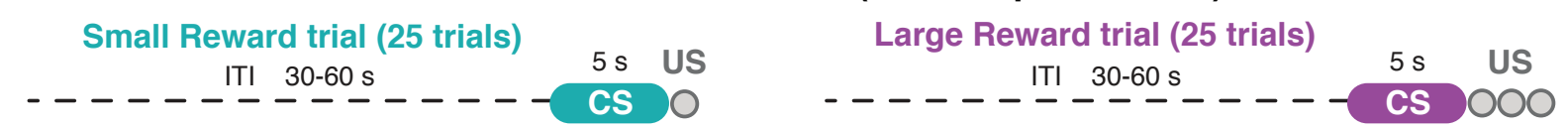

B

Acquisition

Conditioned responding
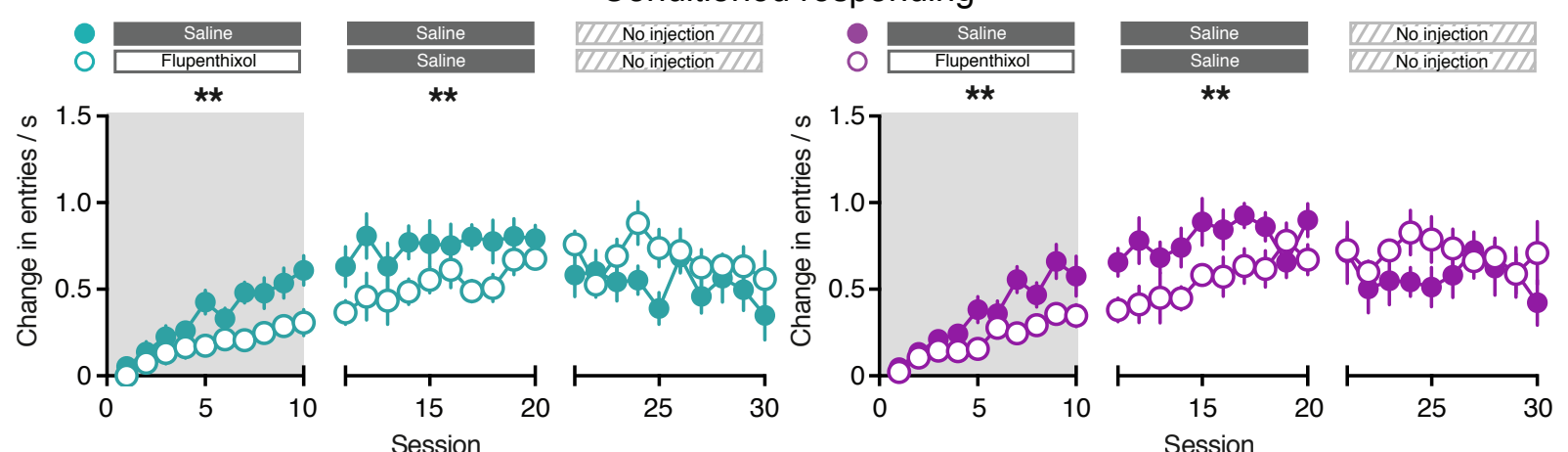

C

Expression
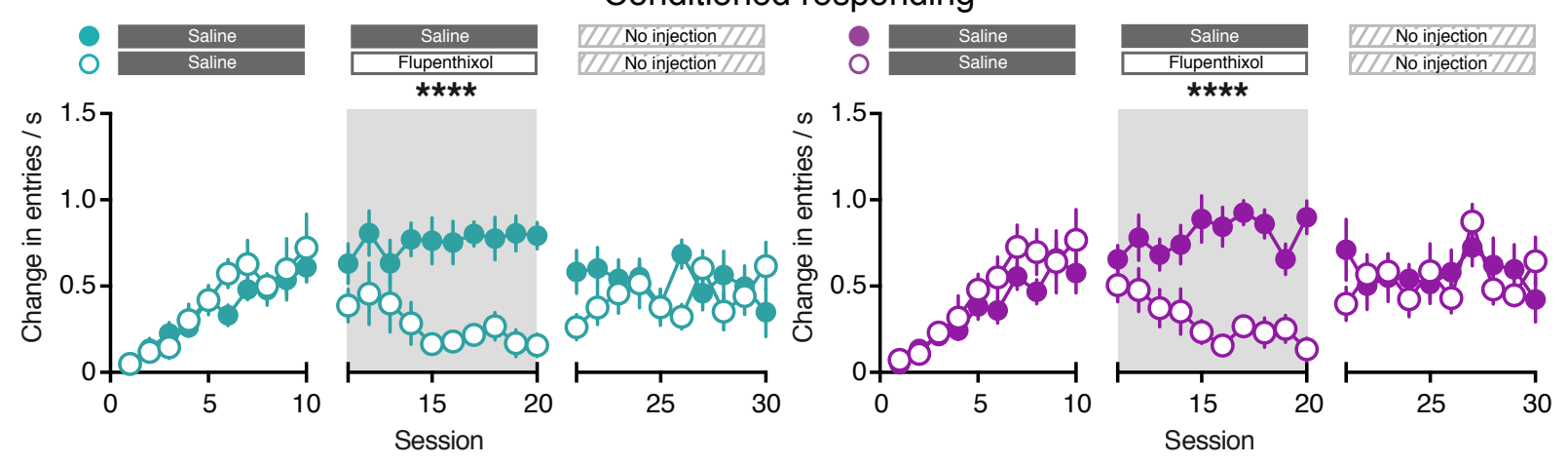

Figure 1. Conditioned responding during the Pavlovian Reward Size task. (A) Pavlovian Reward Size task design. (B) Conditioned responding when flupenthixol is administered during the Acquisition phase (Sessions 1-10; open circles) compared to saline-injected control subjects (closed circles) for Small Reward trials (teal, left) and Large Reward trials (purple, right). (C) Conditioned responding when flupenthixol is administered during the Expression phase (Sessions 11-20; open circles) compared to saline-injected control subjects (closed circles) for Small Reward trials (teal, left) and Large Reward trials (purple, right). ${ }^{* *} p<0.01,{ }^{* * * *} p<0.0001$.

Acquisition phase compared to control rats (Sessions 11-20; treatment effect: $F_{(1,126)}=7.28, p=$ 0.008 ; Fig. 1B). However, there was no difference in conditioned responding between groups in the final ten sessions of training in which no injections were given (Sessions 21-30; treatment effect: $F_{(1,126)}=1.26, p=0.26$; Fig. 1B).

In a separate group of rats that had already undergone ten training sessions we examined how antagonizing the dopamine system regulates conditioned responding during the Expression phase (Fig. 1C). Flupenthixol injections during the Expression phase acutely impaired conditioned responding during both Small and Large Reward trials (Sessions 11-20 three-way mixed-effects analysis; treatment effect: $F_{(1,126)}=18.88, p<0.0001$; session $\mathrm{x}$ treatment effect: 
Pavlovian Reward Rate task (50 trials per session)

\section{Low Rate trial (25 trials)}

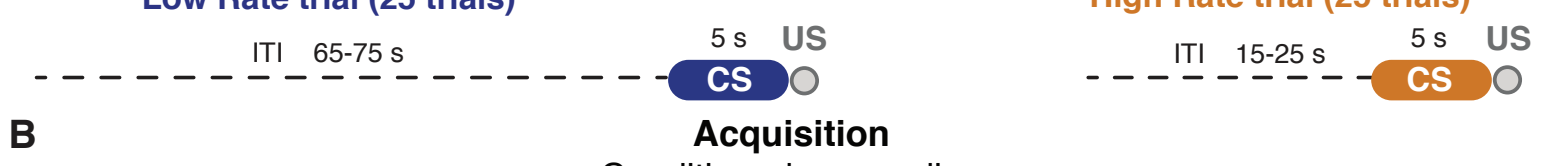

B

Conditioned responding

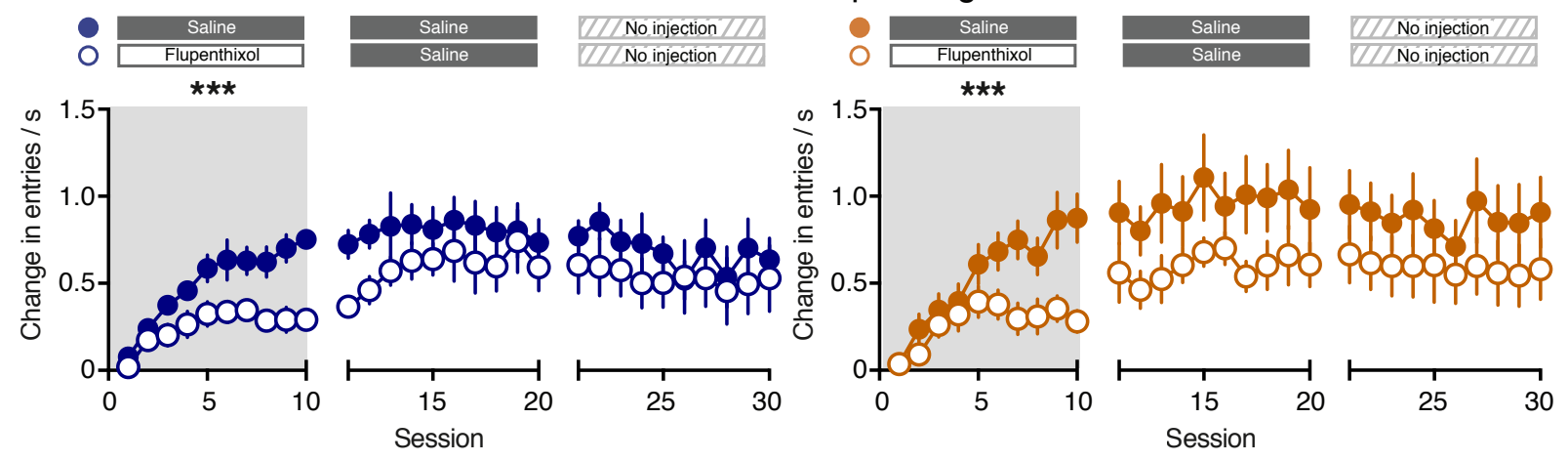

C

Expression

Conditioned responding
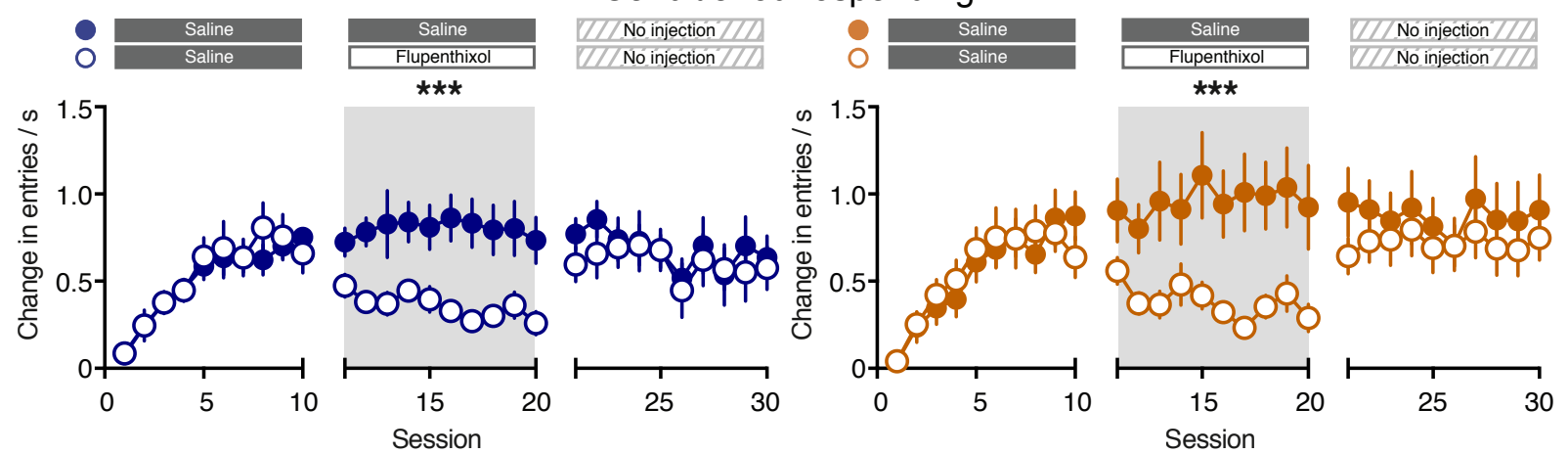

Figure 2. Conditioned responding during the Pavlovian Reward Rate task. (A) Pavlovian Reward Rate task design. (B) Conditioned responding when flupenthixol is administered during the Acquisition phase (Sessions 1-10; open circles) compared to saline-injected control subjects (closed circles) for Low Rate trials (blue, left) and High Rate trials (orange, right). (C) Conditioned responding when flupenthixol is administered during the Expression phase (Sessions 11-20; open circles) compared to saline-injected control subjects (closed circles) for Low Rate trials (blue, left) and High Rate trials (orange, right). ${ }^{* * *} p<0.001$.

$F_{(9,126)}=2.74, p=0.006$; Fig. 1C). There was no effect of treatment during the following ten sessions in which no injections were given (Sessions 21-30; treatment effect: $F_{(1,126)}=0.25, p=$ 0.62; session x treatment effect: $F_{(9,126)}=3.06, p=0.002$; Fig. 1C). Together this indicates that antagonizing dopamine receptors during early training sessions produced both acute and prolonged effects on conditioned responding in rats trained on the Pavlovian Reward Size task. However, flupenthixol treatment in well-trained animals produced only acute effects on conditioned responding. 
In rats trained on the Pavlovian Reward Rate task, there was no difference in conditioned responding between Low and High Rate trials (Sessions 1-10 three-way mixed-effects analysis; reward rate effect: $F_{(1,18)}=0.29, p=0.60$; Fig. 2B), consistent with prior studies (Fonzi et al., 2017; Stelly et al., 2021). Similar to the Pavlovian Reward Size task, antagonizing dopamine receptors during the Acquisition phase impaired conditioned responding in both trial types (Sessions 1-10 three-way mixed-effects analysis; treatment effect: $F_{(1,162)}=12.78, p=0.0005$; session $\mathrm{x}$ treatment effect: $F_{(9,162)}=6.29, p<0.0001$; Fig. 2B). In contrast to the Pavlovian Reward Size task, rats did not display a prolonged decrease in conditioned responding during the next ten sessions of training (Sessions 11-20; treatment effect: $F_{(1,162)}=3.06, p=0.08$; Fig. 2B). However when examining the first session following flupenthixol treatment, conditioned responding was impaired (Session 11 two-way mixed-effects analysis; treatment effect: $F_{(1,14)}=$ 6.11, $p=0.02$; Fig. 2B). Therefore in the Pavlovian Reward Rate task, the deficits in conditioned responding during the Acquisition phase can rapidly reverse when flupenthixol is no longer administered. Antagonizing dopamine receptors during the Expression phase diminished conditioned responding in both Low and High Rate trials (Sessions 11-20 three-way mixedeffects analysis; treatment effect: $F_{(1,162)}=11.90, p=0.0007$; Fig. 2C), though there was no difference between treatment groups in the next ten training sessions following flupenthixol administration (Sessions 21-30; treatment effect: $F_{(1,162)}=0.47, p=0.49$; Fig. 2C). These results illustrate that antagonizing dopamine receptors in rats trained on the Pavlovian Reward Rate task produced acute and rapidly reversible deficits on conditioned responding.

We additionally examined the effects of dopamine receptor antagonism on the latency to enter the food port following the onset of the CS (Fig. 3). In rats trained on the Pavlovian Reward Size task, antagonizing dopamine receptors during the Acquisition phase increased the latency to respond in both Small and Large Reward trials (Sessions 1-10 three-way mixed-effects analysis; treatment effect: $F_{(1,126)}=8.88, p=0.004$; Fig. 3A). However there were no prolonged effects on the latency to respond (Sessions 11-20; treatment effect: $F_{(1,122)}=1.07, p=0.30$; Fig. 3A). Antagonizing dopamine receptors during the Expression phase increased the latency to respond in both trial types (Sessions 11-20 three-way mixed-effects analysis; treatment effect: $F_{(1,126)}=55.73, p<0.0001$; session $\mathrm{x}$ treatment effect: $F_{(9,126)}=4.55, p<0.0001$; Fig. 3B). 
A

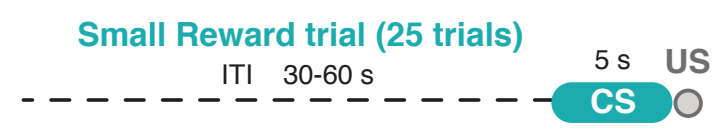

Pavlovian Reward Size task (50 trials per session)

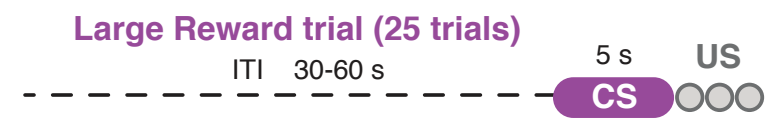

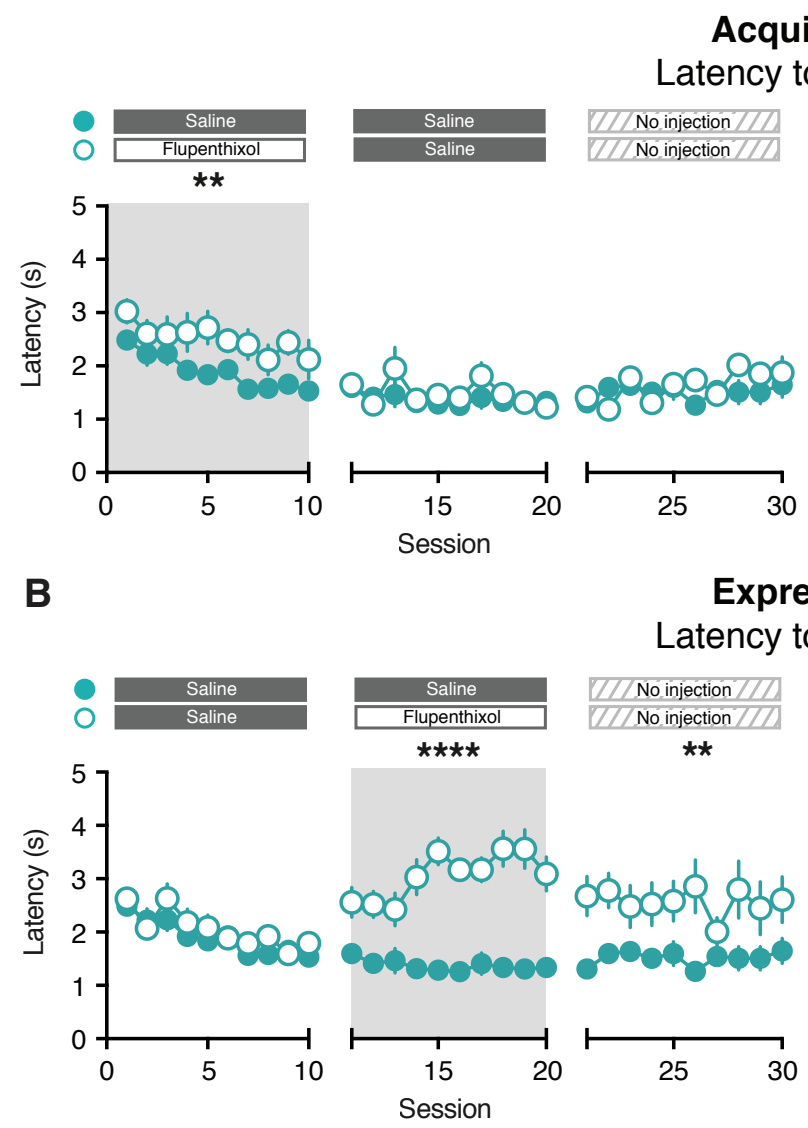

Acquisition

Latency to respond

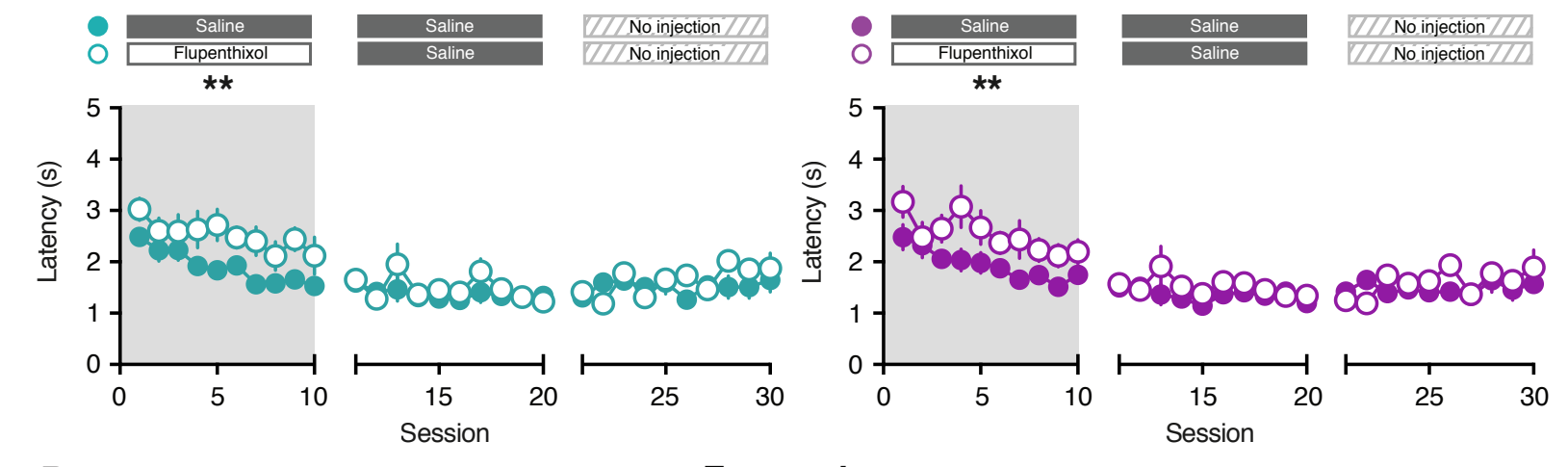

B

Latency to respond

Figure 3. Latency to respond during the Pavlovian Reward Size task. (A) Latency to respond when flupenthixol is administered during the Acquisition phase for Small and Large Reward trials. (B) Latency to respond when flupenthixol is administered during the Expression phase for Small and Large Reward trials. ${ }^{* *} p<0.01,{ }^{* * * *} p<$ 0.0001 .

Additionally, the latency to respond remained elevated in the last set of sessions where no injections were administered (Sessions 21-30; treatment effect: $F_{(1,126)}=7.13, p=0.009$; session $\mathrm{x}$ treatment effect: $F_{(9,126)}=2.26, p=0.02$; Fig. 3B). Together, these results illustrate that perturbing dopamine signaling in well-trained animals produces sustained deficits in the latency to respond, without affecting conditioned responding in rats trained on the Pavlovian Reward Size task (Fig. 1C, Fig. 3B).

In rats trained on the Pavlovian Reward Rate task, antagonizing dopamine receptors during the Acquisition phase also increased the latency to respond in both Low and High Rate trials (Sessions 1-10 three-way mixed-effects analysis; treatment effect: $F_{(1,162)}=5.81, p=0.02$; 


\section{Low Rate trial (25 trials)}

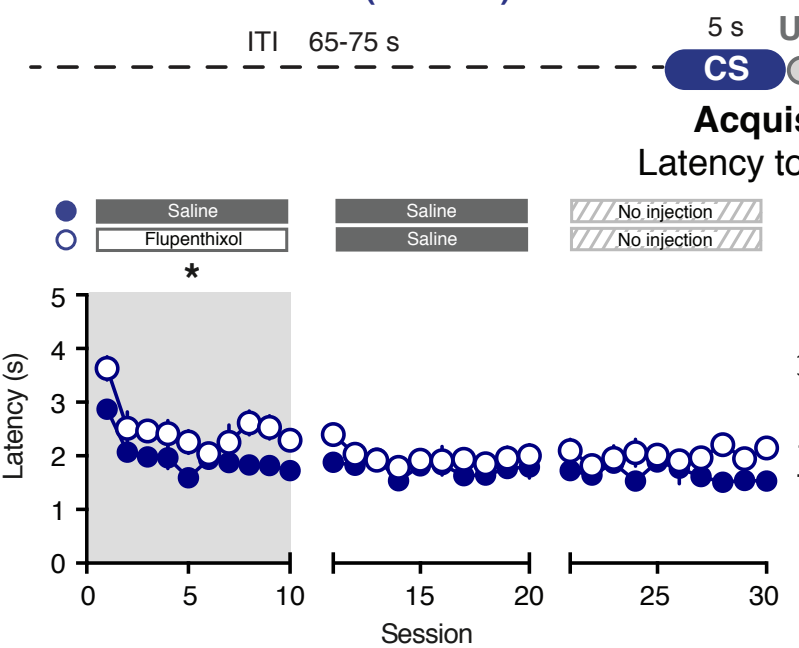

\section{High Rate trial (25 trials)}

B

Expression

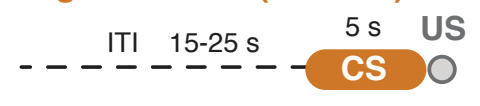

Acquisition

Latency to respond
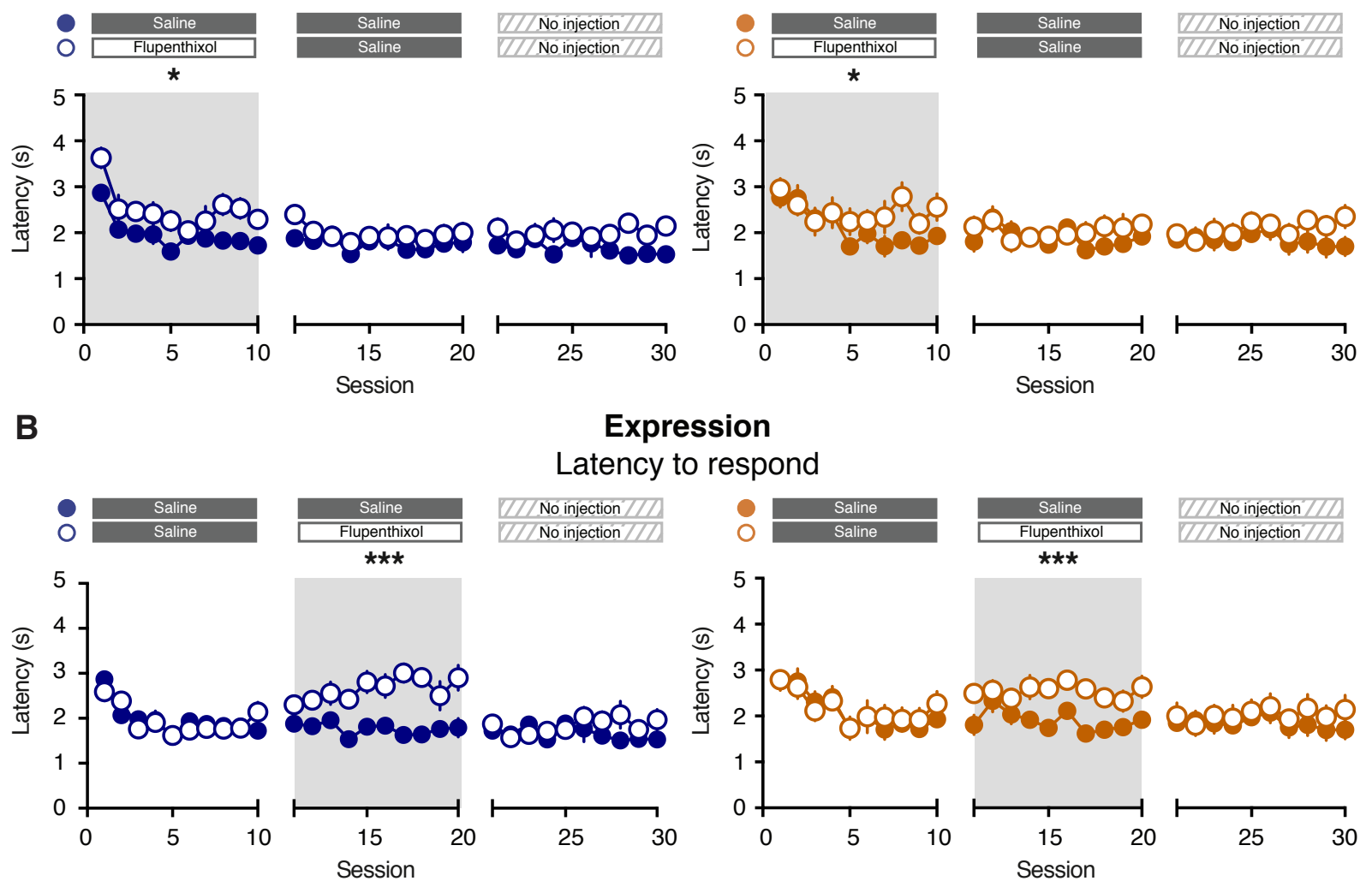

Figure 4. Latency to respond during the Pavlovian Reward Rate task. (A) Latency to respond when flupenthixol is administered during the Acquisition phase for Low and High Rate trials. (B) Latency to respond when flupenthixol is administered during the Expression phase for Low and High Rate trials. ${ }^{*} p<0.05,{ }^{* *} p<0.001$.

Fig. 4A), though there were no differences between groups in the following ten sessions (Sessions 11-20; treatment effect: $F_{(1,162)}=1.22, p=0.27$; Fig. 4A). Similarly, antagonizing dopamine receptors during the Expression phase increased the latency to respond in both trial types (Sessions 11-20; treatment effect: $F_{(1,162)}=15.09, p=0.0001$; session x treatment effect: $F_{(9,162)}=2.42, p<0.01$; Fig. 4B), with no prolonged effects in the following sessions (Sessions 21-30; treatment effect: $F_{(1,162)}=0.79, p=0.37$; Fig. 4B). Collectively, these results demonstrate that the influence of the dopamine system on cue-evoked behavioral responding depends on the phase of training as well as whether the cues denote differences in reward size (Figs. 1,3) or reward rate (Figs. 2,4). 


\section{A

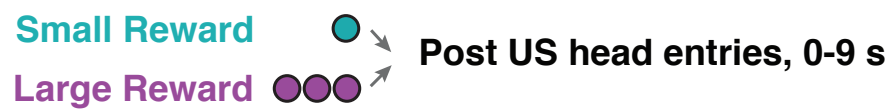

B

Acquisition
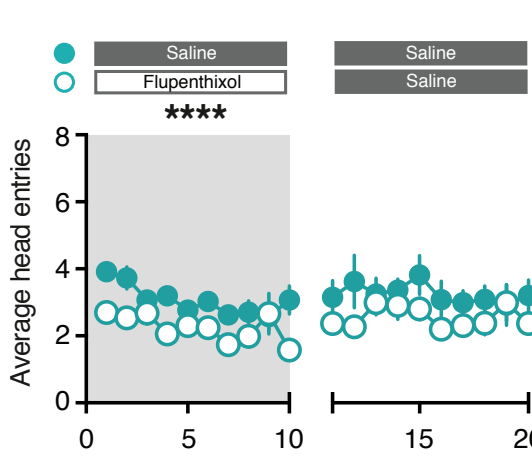

Post US head entries
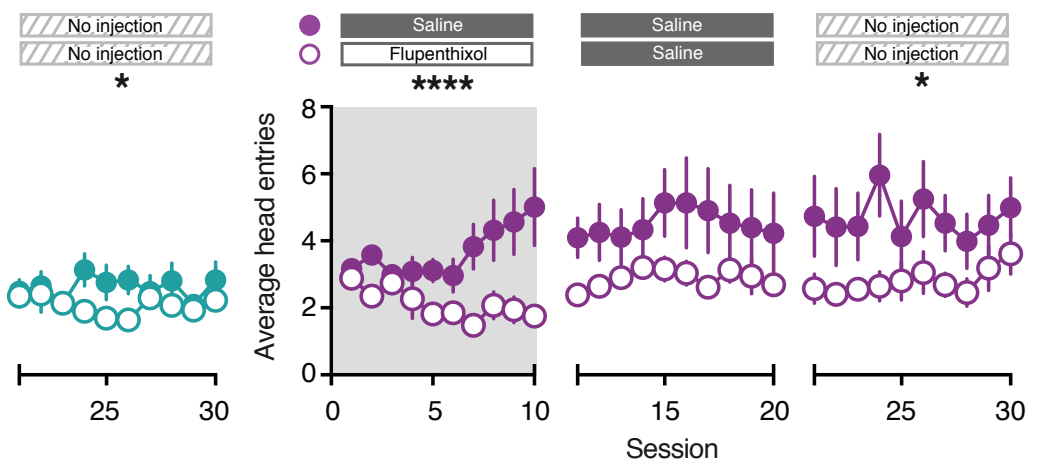

C

Expression

\section{Post US head entries}
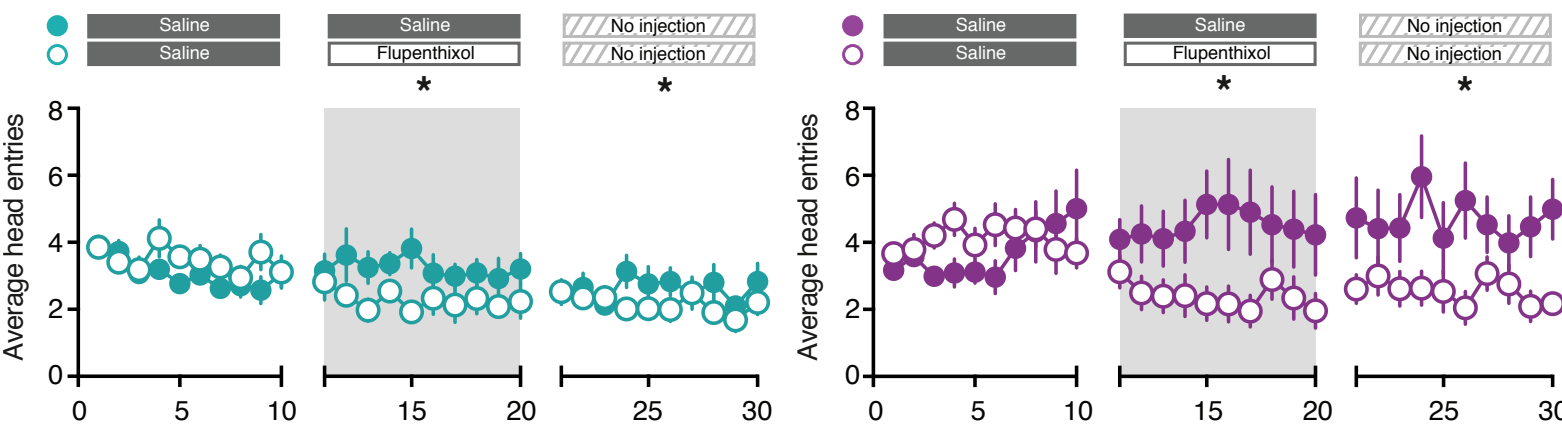

*
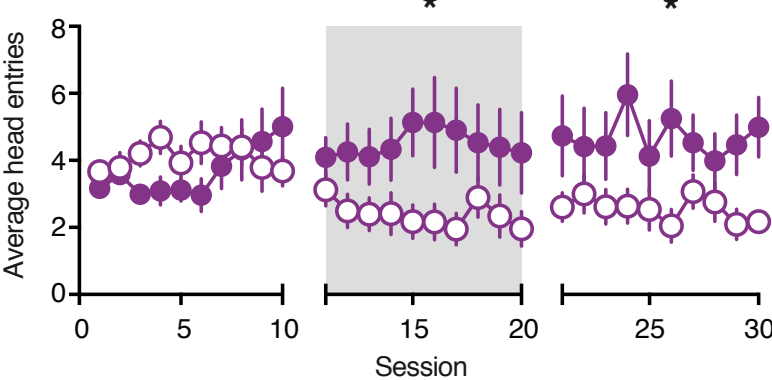

Figure 5. Head entries following reward delivery during the Pavlovian Reward Size task. (A) Post US time window. (B) Post US head entries when flupenthixol is administered during the Acquisition phase for Small and Large Reward trials. (B) Post US head entries when flupenthixol is administered during the Expression phase for Small and Large Reward trials. $* p<0.05, * * * * p<0.0001$.

We next examined behavioral responses following reward delivery by quantifying the number of head entries occurring in the $9 \mathrm{~s}$ after the termination of the CS (Post US; Fig. 5A). Rats perform a greater number of head entries following the delivery of a large reward relative to the delivery of a small reward (Lefner et al., 2022). Flupenthixol administration during the Acquisition phase decreased Post US head entries for both trial types in the Pavlovian Reward Size task (Sessions 1-10 three-way mixed-effects analysis; treatment effect: $F_{(1,126)}=16.73, p<$ 0.0001 ; session $\mathrm{x}$ treatment effect: $F_{(9,126)}=2.11, p=0.03$; three-way interaction effect: $F_{(9,126)}=$ 2.82, $p=0.005$; Fig. 5B). This effect was also observed in the first session following flupenthixol treatment (Session 11 two-way mixed-effects analysis; treatment effect: $F_{(1,14)}=$ $5.48, p=0.03$; Sessions 11-20 three-way mixed-effects analysis; treatment effect: $F_{(1,126)}=3.35$, 


\section{A

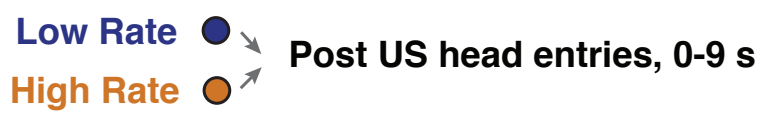

B
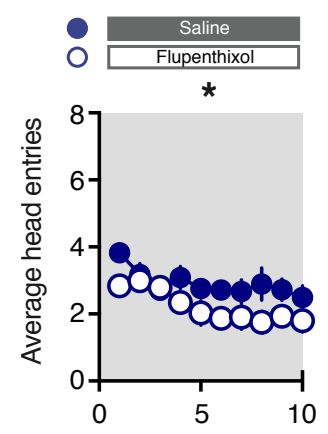

C
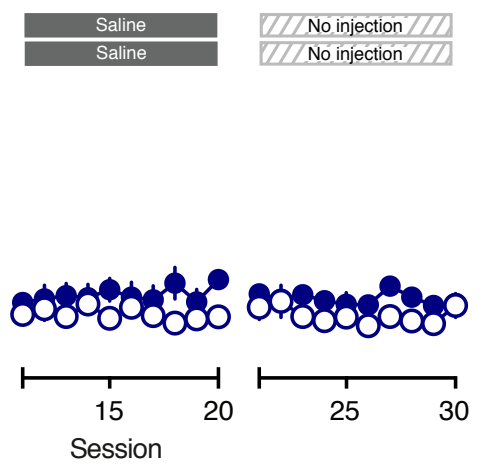

Post US head entries
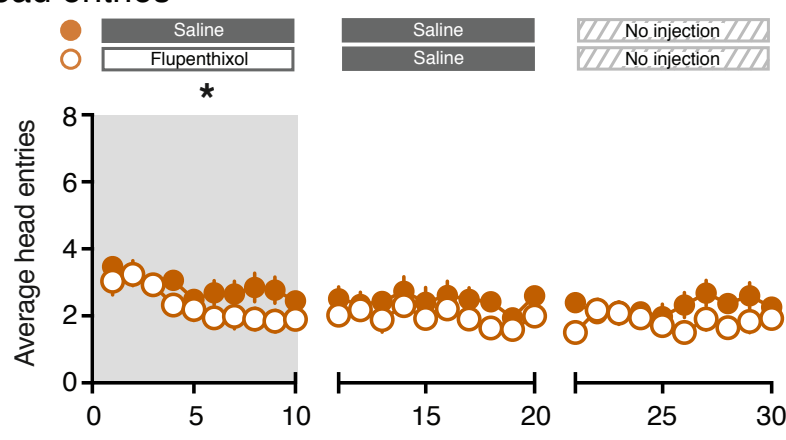

Expression

Post US head entries
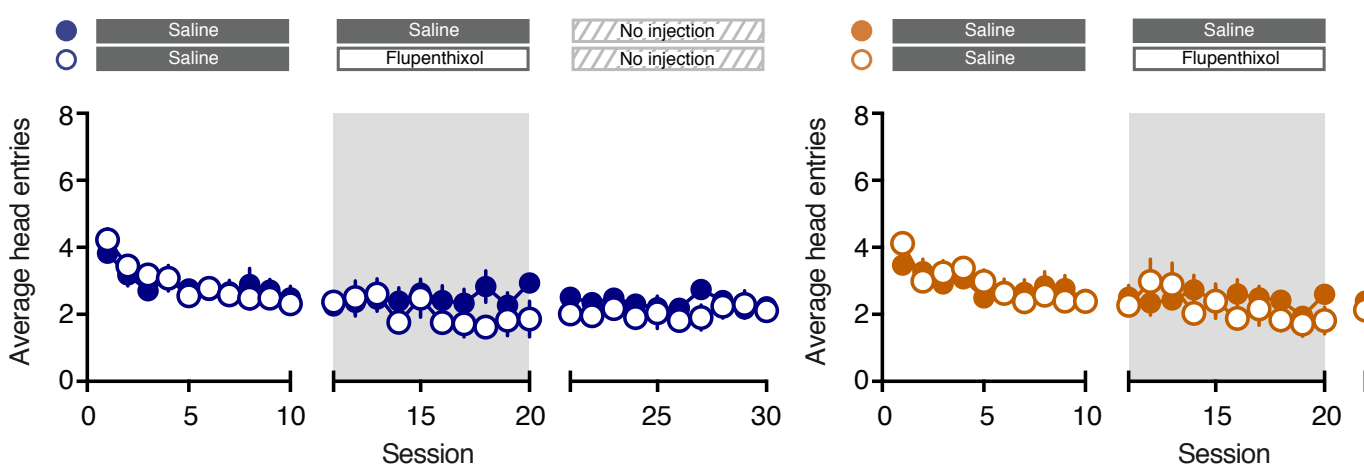

$\frac{\square / \text { No injection } / / 7}{\text { D//No injection } / / 1 /}$

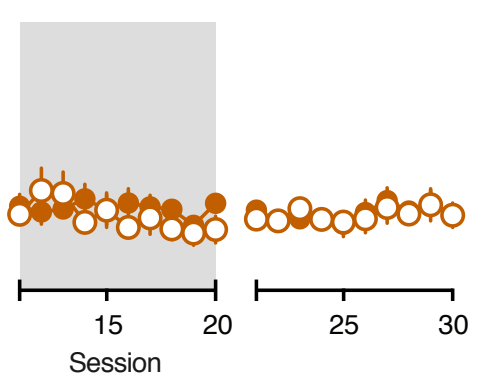

Figure 6. Head entries following reward delivery during the Pavlovian Reward Rate task. (A) Post US time window. (B) Post US head entries when flupenthixol is administered during the Acquisition phase for Low and High Rate trials. (B) Post US head entries when flupenthixol is administered during the Expression phase for Low and High Rate trials. $* p<0.05$.

$p=0.07$; Fig. 5B). Furthermore, the impairments in Post US head entries were also evident during the final ten sessions in which no injections were administered (Sessions 21-30; treatment effect: $F_{(1,126)}=4.47, p=0.04$; Fig. 5B). Antagonizing dopamine receptors during the Expression phase decreased Post US head entries in both trial types (Sessions 11-20 three-way mixed-effects analysis; treatment effect: $F_{(1,126)}=5.23, p=0.02$; Fig. 5C). This effect was also observed in the ten sessions following flupenthixol treatment (Sessions 21-30; treatment effect: $F_{(1,126)}=4.61, p=0.03$; Fig. 5C). These results suggest that dopamine receptor antagonism produces both acute and persistent deficits on Post US head entries in rats trained on the Pavlovian Reward Size task. 
A

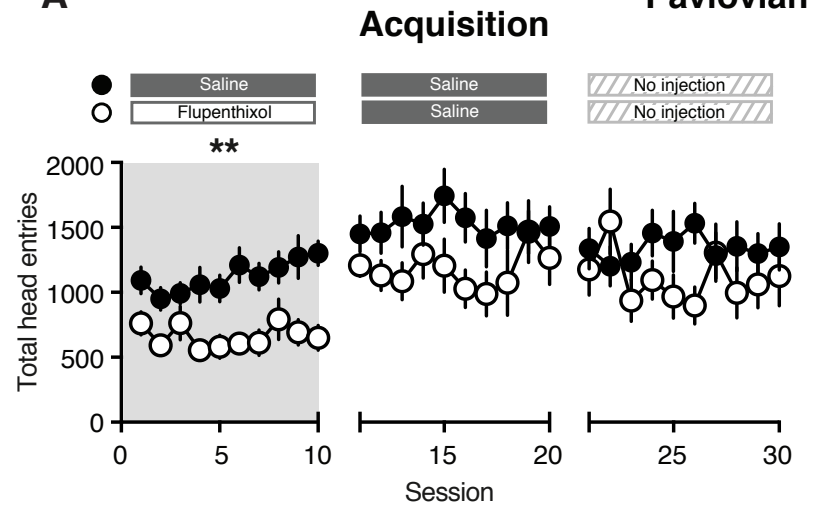

Pavlovian Reward Size

Pavlovian Reward Rate

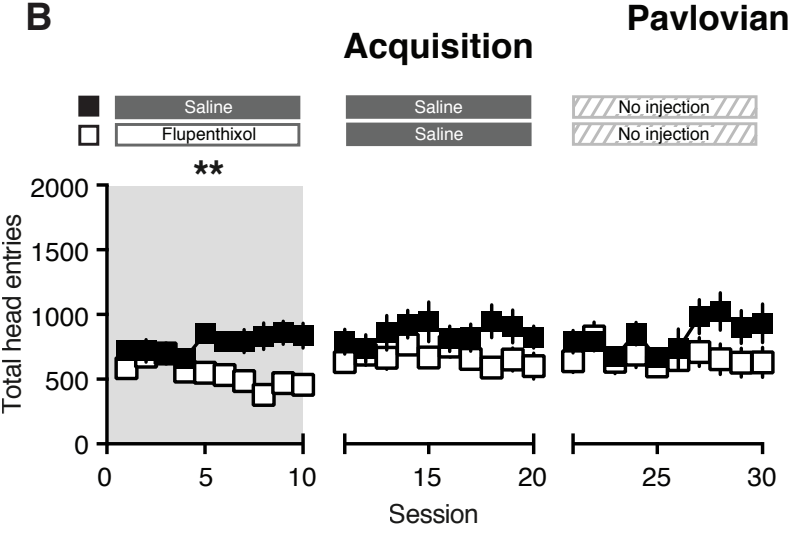

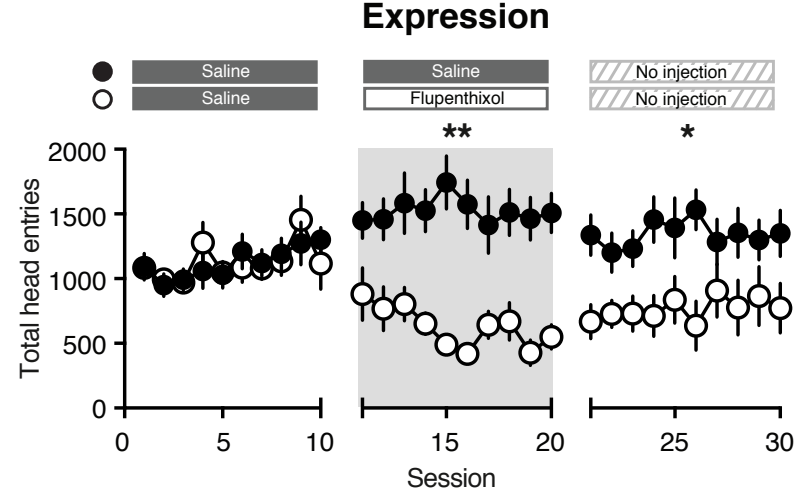

Expression

Expression

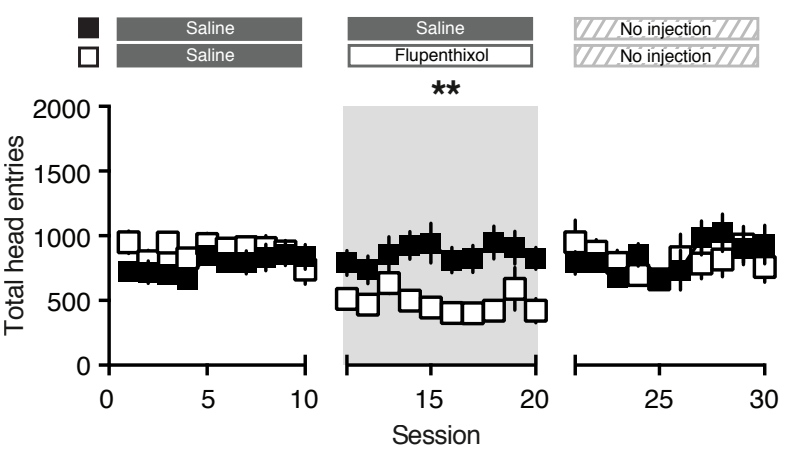

Figure 7. Total head entries. (A) Left; Total head entries when flupenthixol is administered during the Acquisition phase (Sessions 1-10; open circles) compared to saline-injected control subjects (closed circles) for the Pavlovian Reward Size task. Right; Total head entries when flupenthixol is administered during the Expression phase (Sessions 1-10; open circles) compared to saline-injected control subjects (closed circles) for the Pavlovian Reward Size task. (B) Left; Total head entries when flupenthixol is administered during the Acquisition phase (Sessions 1-10; open squares) compared to saline-injected control subjects (closed squares) for the Pavlovian Reward Rate task. Right; Total head entries when flupenthixol is administered during the Expression phase (Sessions 1-10; open squares) compared to saline-injected control subjects (closed squares) for the Pavlovian Reward Rate task. $* p<0.05,{ }^{* *} p<0.01$.

In the Pavlovian Reward Rate task, flupenthixol administration during the Acquisition phase diminished Post US head entries during both Low and High Rate trials (Sessions 1-10 three-way mixed-effects analysis; treatment effect: $F_{(1,162)}=4.29, p=0.04$; Fig. 6A-B), with no effect on the following ten sessions (Sessions 11-20; treatment effect: $F_{(1,162)}=2.28, p=0.13$; Fig. 6B). In contrast, dopamine receptor antagonism during the Expression phase did not produce acute or prolonged effects on Post US head entries (Sessions 11-20; three-way mixedeffects analysis; treatment effect: $F_{(1,162)}=0.64, p=0.42$; Sessions $21-30$; treatment effect: $F_{(1}$, ${ }_{162)}=0.38, p=0.54$; Fig. 6C). These results collectively highlight that the influence of the 


\begin{tabular}{|c|c|c|c|c|c|c|c|c|c|}
\hline \multirow{3}{*}{$\begin{array}{c}\text { Pavlovian } \\
\text { task }\end{array}$} & \multirow{3}{*}{$\begin{array}{l}\text { Flup } \\
\text { effects }\end{array}$} & \multicolumn{4}{|c|}{ CS behavior } & \multirow{2}{*}{\multicolumn{2}{|c|}{$\begin{array}{c}\text { US behavior } \\
\text { Post US head entries }\end{array}$}} & \multirow{2}{*}{\multicolumn{2}{|c|}{ Total head entries }} \\
\hline & & \multicolumn{2}{|c|}{ Conditioned responding } & \multicolumn{2}{|c|}{ Latency to respond } & & & & \\
\hline & & Acquisition & Expression & Acquisition & Expression & Acquisition & Expression & Acquisition & Expression \\
\hline \multirow{2}{*}{$\begin{array}{l}\text { Reward } \\
\text { Size }\end{array}$} & Acute & $\downarrow$ & $\downarrow$ & $\downarrow$ & $\downarrow$ & $\downarrow$ & $\downarrow$ & $\downarrow$ & $\downarrow$ \\
\hline & Post-treatment & $\downarrow$ Prolonged & - & - & - & $\downarrow$ Transient & $\downarrow$ Prolonged & - & $\downarrow$ Prolonged \\
\hline \multirow{2}{*}{$\begin{array}{c}\text { Reward } \\
\text { Rate }\end{array}$} & Acute & $\downarrow$ & $\downarrow$ & $\downarrow$ & $\downarrow$ & $\downarrow$ & - & $\downarrow$ & $\downarrow$ \\
\hline & Post-treatment & $\downarrow$ Transient & - & - & - & - & - & - & - \\
\hline
\end{tabular}

Figure 8. Summary table demonstrating the main effects of treatment administered during the Acquisition phase or the Expression phase on cue- and reward-evoked behaviors during both Pavlovian tasks. Acute effects of flupenthixol on behavior are represented by arrows; sustained post-treatment effects of flupenthixol on behavior are indicated with text.

dopamine system on Post US responding depends on the phase of Pavlovian training as well as the size of the delivered reward (Figs. 5-6).

Prolonged deficits in behavioral responses following dopamine receptor antagonism during early training sessions provides evidence for dopamine's role in Pavlovian learning (Flagel et al., 2011; Sculfort et al., 2016; Roughley and Killcross, 2019; Stelly et al., 2021). However, our current results illustrate that flupenthixol treatment during the Expression phase in the Pavlovian Reward Size task produces sustained deficits in the latency to respond and in Post US head entries (Fig. 3C, Fig. 5C). These flupenthixol-elicited behavioral alterations could arise from a sustained suppression in the number of head entries performed by the subject. We therefore examined how dopamine receptor antagonism impacted the total number of head entries across sessions. Flupenthixol treatment during the Acquisition phase acutely decreased the total number of head entries in rats trained on the Pavlovian Reward Size task (Sessions 1-10 two-way mixed-effects model; treatment effect: $F_{(1,14)}=16.24, p=0.001$; Fig. 7A), with no sustained effect during the following sessions (Sessions 11-20; treatment effect: $F_{(1,14)}=2.54, p$ $=0.13$; Fig. 7A). In contrast, flupenthixol treatment during the Expression phase acutely decreased the total number of head entries (Sessions 11-20 two-way mixed-effects model; treatment effect: $F_{(1,14)}=27.09, p=0.001$; Fig. 7A) and produced sustained deficits in the following sessions without drug injections (Sessions 21-30; treatment effect: $F_{(1,14)}=6.89, p=$ 0.02; Fig. 7A). While dopamine receptor antagonism reduced the total number of head entries in the last ten training sessions of the Pavlovian Reward Size task, this did not impact the CSevoked change in head entries (i.e. conditioned responding; Fig. 1C). In the Pavlovian Reward Rate task, sustained effects on the total number of head entries were not observed when rats 
received flupenthixol injections during the Acquisition phase (Sessions 1-10 two-way mixedeffects model; treatment effect: $F_{(1,18)}=9.47, p=0.007$; Sessions 11-20; treatment effect: $F_{(1,18)}$ $=3.02, \mathrm{p}=0.10$; Fig. 7B) or the Expression phase (Sessions 11-20 two-way mixed-effects model; treatment effect: $F_{(1,18)}=11.85, p=0.003$; Sessions $21-30$; treatment effect: $F_{(1,18)}=$ 0.03, $p=0.86$; Fig. 7B). A summary of the observed effects of dopamine receptor antagonism on cue- and reward-evoked behavioral responses for both Pavlovian tasks can be found in Fig. 8.

\section{Discussion:}

Our findings demonstrate that perturbations of the dopamine system can alter behavioral responding throughout Pavlovian training. Prior research illustrates that dopamine receptor antagonism or optogenetic inhibition of dopamine neurons acutely suppresses conditioned responding (Di Ciano et al., 2001; Saunders and Robinson, 2012; Fraser and Janak, 2017; Heymann et al., 2020; Lee et al., 2020; Morrens et al., 2020). In support, we also found acute effects of dopamine receptor antagonism on both cue- and reward-evoked behavior across Pavlovian tasks. However, we note that the post-reward head entries during the Expression phase of the Pavlovian Reward Rate task were not impacted by flupenthixol injections. This illustrates that the array of behavioral responses exhibited during Pavlovian conditioning are not uniformly regulated by dopamine signaling.

Prior research illustrates that dopamine is involved with learning in Pavlovian conditioning tasks (Flagel et al., 2011; Sculfort et al., 2016; Roughley and Killcross, 2019; Stelly et al., 2021). In support, we find that flupenthixol treatment during early training sessions produced a transient decrease in conditioned responding in the sessions following drug treatment in the Pavlovian Reward Rate task. However, this same manipulation resulted in a prolonged decrease in conditioned responding in the Pavlovian Reward Size task. Paralleling these effects on conditioned responding, flupenthixol treatment produced a sustained decrease in post-reward head entries in the Pavlovian Reward Size task, but no prolonged effects on post-reward head entries in the Pavlovian Reward Rate task. It is unclear what neurobiological mechanism accounts for this differential effect of flupenthixol treatment on behavioral responding between the Pavlovian tasks. However, we note that subjects earn an average of two food pellets per trial 
in the Pavlovian Reward Size task whereas subjects earn an average of one food pellet per trial in the Pavlovian Reward Rate task. We speculate that suppressing dopamine signaling may functionally produce a negative prediction error when the reward is delivered. Therefore, antagonizing dopamine receptors when the expected average reward size is high may then produce longer lasting behavioral effects relative to when the average reward size is low.

The persistent effects of perturbing the dopamine system has been commonly studied when the dopamine receptor antagonist is administered during early training sessions (Flagel et al., 2011; Roughley and Killcross, 2019; Stelly et al., 2020; Stelly et al., 2021). Here, we additionally examined if dopamine receptor antagonism resulted in prolonged disruption of behavior in well-trained animals. Our findings indicate that flupenthixol treatment does not produce long-lasting behavioral effects in well-trained subjects undergoing the Pavlovian Reward Rate task. In contrast, flupenthixol treatment during the Expression phase produced persistent deficits on the latency to respond, the post-reward head entries, and the total head entries in the Pavlovian Reward Size task. This collectively highlights the presence of critical periods in which the dopamine system exerts control over behavioral responding during Pavlovian learning depends upon what the cues are signaling.

The Pavlovian tasks in our study used audio cues which elicit goal-tracking behavior. Prior studies have found that dopamine receptor antagonism can acutely disrupt goal-tracking behavior (Flagel et al., 2011; Wassum et al., 2011; Sculfort et al., 2016; Roughley and Killcross, 2019; Stelly et al., 2021). Our results extend on these findings as we demonstrate both acute and sustained effects of flupenthixol treatment on conditioned responding in the Pavlovian Reward Size task. However, it is important to acknowledge that the sustained effects of dopamine receptor antagonism depends on the information signaled by the cues as well as the modality of cue utilized. For example in a task where subjects can perform both sign- and goal-tracking responses, prolonged effects of dopamine receptor antagonism are not observed on goal-tracking behavior (Flagel et al., 2011). Therefore, one must exercise caution when comparing the results between Pavlovian studies without considering what types of behavioral responding is elicited by the cues. 
Our experimental approach used flupenthixol which is a non-selective dopamine receptor antagonist. As such it is unclear whether the observed effects on behavior are primarily regulated by D1- or D2-type receptors. Prior research illustrates that administration of either a D1 or D2 receptor antagonist decreases goal-tracking behavior (Lopez et al., 2015; Fraser et al., 2016; Roughley and Killcross, 2019; Khoo et al., 2021), but see (Horvitz, 2001; Eyny and Horvitz, 2003). Future studies are necessary to determine the role of D1- and D2-type receptors in the acquisition and expression of behavioral responding in the Reward Size and Reward Rate Pavlovian tasks. By utilizing systemic injections in the current work, it remains unclear which neural regions are mediating the observed behavioral effects. Prior studies have implicated a role for dopamine signaling in the ventral striatum, as local injections of flupenthixol into the nucleus accumbens core decreased conditioned responding during sessions in which the antagonist was administered (Di Ciano et al., 2001; Saunders and Robinson, 2012; Fraser and Janak, 2017; Stelly et al., 2021). Prolonged deficits on conditioned responding are observed for a least one session following local injections of flupenthixol into the nucleus accumbens core, the nucleus accumbens shell, or the ventral lateral striatum (Stelly et al., 2020; Stelly et al., 2021). Therefore further experiments are needed to determine where dopamine is mediating the acute and prolonged effects on behavioral responses during different Pavlovian tasks.

We found no differences in conditioned responding between trial types in either Pavlovian task. However, recordings of dopamine release in animals trained on these tasks demonstrate that reward-evoked dopamine release signals differences in reward size during early training sessions and cue-evoked dopamine release signals differences in reward rate and reward size in well-trained subjects (Fonzi et al., 2017; Lefner et al., 2022). These findings collectively illustrate that a difference in cue-evoked dopamine release is not driving a corresponding difference in conditioned responding between trials. Rather our prior results demonstrate that when the dopamine response to a given cue changes in well-trained subjects, the animal then exhibits a corresponding update in conditioned responding toward that cue (Fonzi et al., 2017). Future studies are needed to determine if updates in cue-evoked dopamine release similarly controls changes in the latency to respond or post-reward head entries as well as if these behavioral effects are observed across sexes. In sum, our data demonstrates that dopamine's 
control over responding during Pavlovian conditioning depends on (i) what behavior is being studied, (ii) prior training experience, and (iii) the information signaled by the cues.

\section{Funding and Disclosure:}

This work was supported by National Institutes of Health grants DA033386 (MJW) and DA042362 (MJW). The authors declare no conflict of interest.

\section{Author Contributions:}

MJL, CES, KMF, and HZ performed the experiments and analyzed the data. MJW designed the experiments. MJL and MJW wrote the manuscript.

\section{References:}

Coddington LT, Dudman JT (2018) The timing of action determines reward prediction signals in identified midbrain dopamine neurons. Nat Neurosci 21:1563-1573.

Day JJ, Roitman MF, Wightman RM, Carelli RM (2007) Associative learning mediates dynamic shifts in dopamine signaling in the nucleus accumbens. Nat Neurosci 10:1020-1028.

Di Ciano P, Cardinal RN, Cowell RA, Little SJ, Everitt BJ (2001) Differential involvement of NMDA, AMPA/kainate, and dopamine receptors in the nucleus accumbens core in the acquisition and performance of pavlovian approach behavior. J Neurosci 21:9471-9477.

Eyny YS, Horvitz JC (2003) Opposing roles of D1 and D2 receptors in appetitive conditioning. J Neurosci 23:1584-1587.

Fiorillo CD, Tobler PN, Schultz W (2003) Discrete coding of reward probability and uncertainty by dopamine neurons. Science 299:1898-1902.

Flagel SB, Clark JJ, Robinson TE, Mayo L, Czuj A, Willuhn I, Akers CA, Clinton SM, Phillips PE, Akil H (2011) A selective role for dopamine in stimulus-reward learning. Nature 469:53-57.

Fonzi KM, Lefner MJ, Phillips PEM, Wanat MJ (2017) Dopamine Encodes Retrospective Temporal Information in a Context-Independent Manner. Cell Rep 20:1765-1774.

Fraser KM, Janak PH (2017) Long-lasting contribution of dopamine in the nucleus accumbens core, but not dorsal lateral striatum, to sign-tracking. Eur J Neurosci 46:2047-2055.

Fraser KM, Haight JL, Gardner EL, Flagel SB (2016) Examining the role of dopamine D2 and D3 receptors in Pavlovian conditioned approach behaviors. Behav Brain Res 305:87-99. 
Gan JO, Walton ME, Phillips PE (2010) Dissociable cost and benefit encoding of future rewards by mesolimbic dopamine. Nat Neurosci 13:25-27.

Hart AS, Clark JJ, Phillips PEM (2015) Dynamic shaping of dopamine signals during probabilistic Pavlovian conditioning. Neurobiol Learn Mem 117:84-92.

Heymann G, Jo YS, Reichard KL, McFarland N, Chavkin C, Palmiter RD, Soden ME, Zweifel LS (2020) Synergy of Distinct Dopamine Projection Populations in Behavioral Reinforcement. Neuron 105:909-920 e905.

Horvitz JC (2001) The effects of D1 and D2 receptor blockade on the acquisition and expression of a conditioned appetitive response. Appetite 37:119-120.

Khoo SY, Uhrig A, Samaha AN, Chaudhri N (2021) Effects of dopamine receptor antagonism and amphetamine-induced psychomotor sensitization on sign- and goal-tracking after extended training. Behav Brain Res 407:113238.

Lee K, Claar LD, Hachisuka A, Bakhurin KI, Nguyen J, Trott JM, Gill JL, Masmanidis SC (2020) Temporally restricted dopaminergic control of reward-conditioned movements. Nat Neurosci 23:209-216.

Lefner MJ, I. DM, Wanat MJ (2022) Sex differences in behavioral responding and dopamine release during Pavlovian learning. BioRxiv. https://doi.org/10.1101/2021.10.04.463059

Lopez JC, Karlsson RM, O'Donnell P (2015) Dopamine D2 Modulation of Sign and Goal Tracking in Rats. Neuropsychopharmacology 40:2096-2102.

Morrens J, Aydin C, Janse van Rensburg A, Esquivelzeta Rabell J, Haesler S (2020) Cue-Evoked Dopamine Promotes Conditioned Responding during Learning. Neuron 106:142-153 e147.

Phillips PE, Walton ME, Jhou TC (2007) Calculating utility: preclinical evidence for cost-benefit analysis by mesolimbic dopamine. Psychopharmacology (Berl) 191:483-495.

Roesch MR, Calu DJ, Schoenbaum G (2007) Dopamine neurons encode the better option in rats deciding between differently delayed or sized rewards. Nat Neurosci 10:1615-1624.

Roughley S, Killcross S (2019) Differential involvement of dopamine receptor subtypes in the acquisition of Pavlovian sign-tracking and goal-tracking responses. Psychopharmacology (Berl) 236:1853-1862.

Salamone JD, Correa M (2012) The mysterious motivational functions of mesolimbic dopamine. Neuron 76:470-485.

Saunders BT, Robinson TE (2012) The role of dopamine in the accumbens core in the expression of Pavlovian-conditioned responses. Eur J Neurosci 36:2521-2532.

Schultz W, Dayan P, Montague PR (1997) A neural substrate of prediction and reward. Science 275:1593-1599.

Sculfort SA, Bartsch D, Enkel T (2016) Dopamine antagonism does not impair learning of Pavlovian conditioned approach to manipulable or non-manipulable cues but biases responding towards goal tracking. Behav Brain Res 314:1-5.

Stelly CE, Tritley SC, Rafati Y, Wanat MJ (2020) Acute Stress Enhances Associative Learning via Dopamine Signaling in the Ventral Lateral Striatum. J Neurosci 40:4391-4400.

Stelly CE, Girven KS, Lefner MJ, Fonzi KM, Wanat MJ (2021) Dopamine release and its control over early Pavlovian learning differs between the NAc core and medial NAc shell. Neuropsychopharmacology 46:1780-1787. 
Tobler PN, Fiorillo CD, Schultz W (2005) Adaptive coding of reward value by dopamine neurons. Science 307:1642-1645.

Wassum KM, Ostlund SB, Balleine BW, Maidment NT (2011) Differential dependence of Pavlovian incentive motivation and instrumental incentive learning processes on dopamine signaling. Learn Mem 18:475-483. 